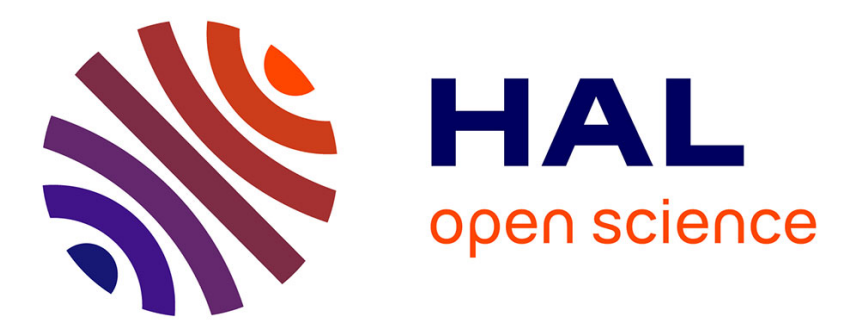

\title{
Dimensioning of Cable-Driven Parallel Robot Actuators, Gearboxes and Winches according to the Twist Feasible Workspace
}

\author{
Lorenzo Gagliardini, Stéphane Caro, Marc Gouttefarde
}

\section{To cite this version:}

Lorenzo Gagliardini, Stéphane Caro, Marc Gouttefarde. Dimensioning of Cable-Driven Parallel Robot Actuators, Gearboxes and Winches according to the Twist Feasible Workspace. CASE: Conference on Automation Science and Engineering, IEEE, Aug 2015, Göteborg, Sweden. pp.99-105, 10.1109/CoASE.2015.7294046 . hal-01941620

\author{
HAL Id: hal-01941620 \\ https://hal.science/hal-01941620
}

Submitted on 1 Dec 2018

HAL is a multi-disciplinary open access archive for the deposit and dissemination of scientific research documents, whether they are published or not. The documents may come from teaching and research institutions in France or abroad, or from public or private research centers.
L'archive ouverte pluridisciplinaire HAL, est destinée au dépôt et à la diffusion de documents scientifiques de niveau recherche, publiés ou non, émanant des établissements d'enseignement et de recherche français ou étrangers, des laboratoires publics ou privés. 


\title{
Dimensioning of Cable-Driven Parallel Robot Actuators, Gearboxes And Winches According To The Twist Feasible Workspace
}

\author{
Lorenzo Gagliardini $^{1}$, Stéphane Caro ${ }^{2}$ and Marc Gouttefarde ${ }^{3}$
}

\begin{abstract}
Cable Driven Parallel Robots (CDPRs) are a particular class of parallel robots whose legs consist of cables. CDPRs are composed of several components, e.g. winches, pulleys and actuators. The design of a CDPR requires the dimensioning of all these components, according to the task to be performed. The dimensioning of the actuators, the gearboxes and the winches are strictly related to the performances of the CDPR in terms of the platform static and kinematic equilibrium.

This paper introduces a new tool, the so called Twist Feasible Workspace (TFW), built in order to analyze the workspace of the platform twists. A pose is said to be twist feasible if the platform of the CDPR can assume a given range of linear and rotational velocities while satisfying the cable speed limits imposed by the actuators and the transmission systems. The size of the TFW is used as an optimization criterion for the dimensioning of the actuators and the winches.
\end{abstract}

\section{INTRODUCTION}

During the last decades, the necessity to perform industrial operations over products of large dimensions promoted the investigation of a new category of robots, the Cable-Driven Parallel Robots (CDPRs), whose legs consist of cables. One side of the cables is connected to a mobile platform while the other side is connected to a base frame. The cable connection points on the base are defined hereafter as exit points. Fig. 1 shows an example of CDPR design, realised in the framework of the IRT Jules Verne's CAROCA project.

Several CDPRs have been designed for a wide range of applications, including the displacement of heavy charges, [1],[2] and the painting of large products [3],[4],[5]. Other possible applications are: the shooting of sport events [6], the rehabilitation of injured people [7] and search and rescue operations [8]. All the previous applications take advantage of the qualities of CDPRs, such as their wide workspace, their reduced inertia and their high payload to weight ratio,

However, CDPRs are characterized by several drawbacks: cable interferences, cable collisions with the surrounding environment and the influence of the non-rigid nature of the cables over the platform positioning precision. Moreover, since the cables cannot push the platform, its static

This work is supported by the IRT Jules Verne, Bouguenais, France (Project CAROCA, Evaluation des CApacités de la RObotique à CÂbles dans un contexte industriel)

${ }^{1}$ L. Gagliardini is with IRT Jules Verne, Chemin du Chaffault, 44340, Bouguenais, France, lorenzo.gagliardini@irt-jules-verne.fr

${ }^{2}$ S. Caro is with CNRS-IRCCyN, 1, rue de la Noë, 44321, Nantes Cedex 03, France, Stephane.Caro@irccyn.ec-nantes.fr

${ }^{3} \mathrm{M}$. Gouttefarde is with the Laboratoire d'Informatique, de Robotique et de Micro-électronique de Montpellier (LIRMM-CNRS-UM), 161, rue Ada, 34392, Montpellier Cedex 05, France, marc.gouttefarde@lirmm.fr

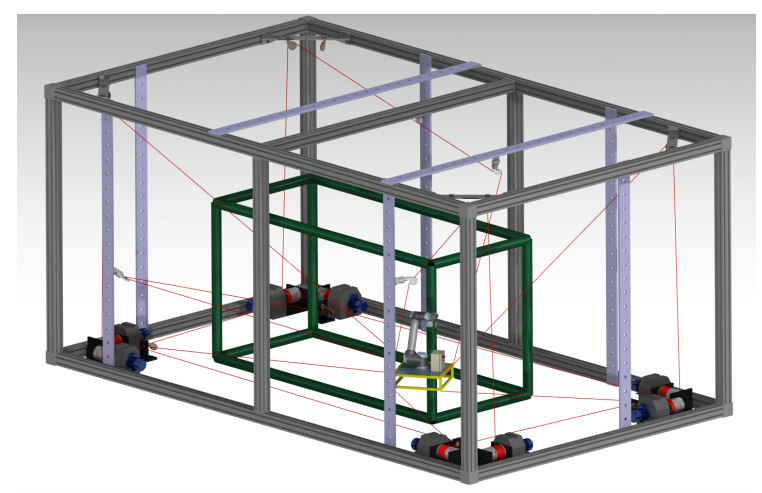

Fig. 1. Example of a CDPR design. The robot shown in the picture is a concept created in the framework of the CAROCA project, whose intent is the painting of tubular structures.

equilibrium is strictly dependant to the cable layout and the cable tension limits.

Considering the cables sufficiently resistant to bare the platform payload, the maximum tension transmitted on the cables depends mostly on the characteristics of the actuators, such as their nominal torque. The actuators influence also the twist that the CDPR platform can attain, being the nominal speed of the actuators proportional to the coiling/uncoiling speed of the cables.

Both the static and the kinematic aspects should be taken into account during the desgin of CDPRs. The static equilibrium of the platform can be analysed using the Wrench Feasible Workspace (WFW) [9]. By definition, the WFW is the set of wrench feasible platform poses. A pose is wrench feasible when the cables can bare a set of external wrenches and the cable tensions stay in between the given cable tension limits. In this paper, the concept of wrench feasibility will be extended to the platform kinematic analysis, introducing the so called Twist Feasible Workspace (TFW). The TFW is the set of twist feasible platform poses. A pose is twist feasible when the actuators, according to their nominal speed, are able to move the platform within a given range of linear and rotational velocities.

A procedure aiming at dimensioning the actuators and the winches of the CDPR has been developed. The design problem has been formulated as an optimization problem maximizing the size of the intersection between the TFW and the WFW.

This paper is organized as follows. Section II introduces the geometric, kinematic and static models used in this paper. Section III introduces the concept of TFW. The TFW is 
used as part of the design process described in Section IV. Section V provides an illustrative example and Section VI concludes this paper.

\section{GEOMETRIC, STATIC AND KINEMATIC ROBOT MODELS}

A CDPR consists of a mobile platform connected to a fixed base by $m$ cables. The pose of the platform, $\mathbf{p}$, is composed of the Cartesian coordinates of the platform Centre of Mass (CoM), t $\mathbf{t}$, and the platform roll, pitch and yaw angles, $\phi, \theta$ and $\psi$. The platform orientation angles are collected in the vector $\boldsymbol{\Omega}=[\phi, \theta, \psi]^{\mathrm{T}}$. The pose is described with respect to an absolute reference frame, $\mathscr{F}_{b}$, of origin $O_{b}$ and axes $\mathbf{x}_{b}, \mathbf{y}_{b}$ and $\mathbf{z}_{b}$.

The Cartesian coordinate vectors $\mathbf{a}_{i}^{b}, i=1, \ldots, m$ describe the positions of the exit points, $A_{i}, i=1, \ldots, m$, with respect to frame $\mathscr{F}_{b}$. The Cartesian coordinate vectors $\mathbf{b}_{i}^{p}, i=1, \ldots, m$ represent the positions of the platform-cable connection points, $B_{i}, i=1, \ldots, m$, with respect to a local reference frame, $\mathscr{F}_{p}$, of origin $O_{p}$ and axes $\mathbf{x}_{p}, \mathbf{y}_{p}$ and $\mathbf{z}_{p}$. This local reference frame is attached to the mobile platform.

\section{A. Geometric model}

The geometric model of a generic CDPR is shown in Fig. 2. The chain closure equations of the cable vectors, defined with respect to $\mathscr{F}_{b}$, compose the geometric model of the CDPR:

$$
\mathbf{l}_{i}^{b}=\mathbf{a}_{i}^{b}-\mathbf{t}-\mathbf{R b}_{i}^{p}, i=1, \ldots, m
$$

where $\mathbf{R}$ denotes the orientation matrix of the platform:

$$
\begin{aligned}
\mathbf{R} & =\mathbf{R}_{z}(\phi) \mathbf{R}_{y}(\theta) \mathbf{R}_{x}(\psi)= \\
& =\left[\begin{array}{ccc}
c \phi c \theta & c \phi s \theta s \psi-s \phi c \psi & c \phi s \theta c \psi+s \phi s \psi \\
s \phi c \theta & s \phi s \theta s \psi+c \phi c \psi & s \phi s \theta c \psi-c \phi s \psi \\
-s \theta & c \theta s \psi & c \theta c \psi
\end{array}\right]
\end{aligned}
$$

The cable unit vectors can be derived directly by the geometric model:

$$
\mathbf{d}_{i}^{b}=\frac{\mathbf{l}_{i}^{b}}{\left\|\mathbf{l}_{i}^{b}\right\|_{2}}, i=1, \ldots, m
$$

where $\left\|\mathbf{l}_{i}^{b}\right\|_{2}$ is the Euclidean norm of $\mathbf{l}_{i}^{b}$.

\section{B. Static model}

The static equilibrium of the CDPR platform is described by the following equation:

$$
\mathbf{W} \boldsymbol{\tau}+\mathbf{w}_{e}=0
$$

where $\mathbf{W}$ is the so called wrench matrix, whose columns are the unit wrenches $\hat{\mathbf{w}}_{i}$ exerted by the cables on the platform:

$$
\mathbf{W}=\left[\begin{array}{cccc}
\mathbf{d}_{1}^{b} & \mathbf{d}_{2}^{b} & \ldots & \mathbf{d}_{m}^{b} \\
\mathbf{R} \mathbf{b}_{1}^{p} \times \mathbf{d}_{1}^{b} & \mathbf{R b}_{2}^{p} \times \mathbf{d}_{2}^{b} & \ldots & \mathbf{R b}_{m}^{p} \times \mathbf{d}_{m}^{b}
\end{array}\right]
$$

The cable tensions $\tau_{i}, i=1, \ldots, m$ are collected in the vector $\boldsymbol{\tau}=\left[\tau_{1}, \ldots, \tau_{m}\right]^{\mathrm{T}}$. The external wrench acting on the platform, $\mathbf{w}_{e}$, is defined as follows:

$$
\mathbf{w}_{e}=\left[\mathbf{f}^{\mathrm{T}}, \mathbf{m}^{\mathrm{T}}\right]^{\mathrm{T}}=\left[f_{x}, f_{y}, f_{z}, m_{x}, m_{y}, m_{z}\right]^{\mathrm{T}}
$$

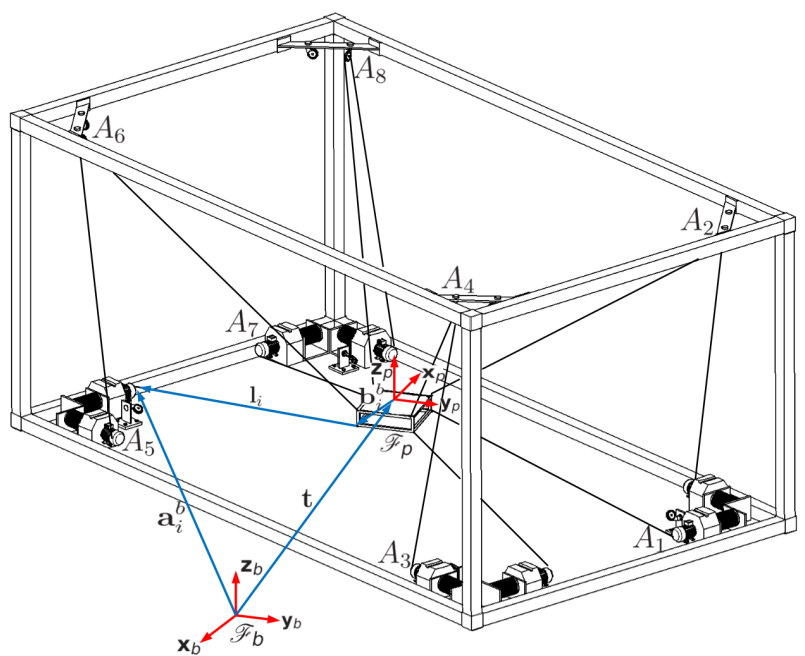

Fig. 2. Geometric description of an example of fully constrained CDPR.

The components of the external force vector, $\mathbf{f}$, as well as the components of the external moment vector, $\mathbf{m}$, are bounded:

$$
\begin{gathered}
f_{\min } \leq f_{x}, f_{y}, f_{z} \leq f_{\max } \\
m_{\min } \leq m_{x}, m_{y}, m_{z} \leq m_{\max }
\end{gathered}
$$

\section{Kinematic model}

The kinematic model of the CDPR describes the twist of the platform in terms of the cable coiling/uncoiling velocities:

$$
\mathbf{J i}-\dot{\mathbf{p}}=\mathbf{J} \mathbf{i}-\left[\begin{array}{c}
\dot{\mathbf{t}} \\
\dot{\omega}
\end{array}\right]=0
$$

where $\dot{t}$ is the vector of the platform linear velocity, $\boldsymbol{\omega}$ is the vector of the platform angular velocity and $\mathrm{i}$ is the vector of the cable velocities, $\mathrm{i}=\left[\dot{l}_{1}, \ldots, l_{m}\right] . \mathbf{J}$ is the Jacobian matrix of the CDPR. The columns of the Jacobian matrix represent the twist $\hat{\dot{\mathbf{p}}}_{i}$ associated to each cable.

Considering the duality of the geometric-static model, the static equilibrium of the platform can be rewritten as:

$$
\boldsymbol{\tau}=\mathbf{J}^{\mathrm{T}} \mathbf{w}_{e}
$$

Comparing (4) and (10) we observe that:

$$
\mathbf{J}^{\mathrm{T}} \mathbf{w}_{e}=-\mathbf{W}^{\dagger} \mathbf{w}_{e}
$$

The Jacobian matrix can be computed as:

$$
\mathbf{J}=-\mathbf{W}^{\dagger^{T}}
$$

Consequently, the kinematic model in (9) can be rewritten as:

$$
\mathbf{W}^{\dagger^{\mathrm{T}}} \mathbf{i}+\dot{\mathbf{p}}=0
$$

where $\mathbf{W}^{\dagger}$ is the Moore-Penrose pseudo-inverse. Since the system is not square, an infinite number of solutions can be associated to Eq. 13. The pseudo-inverse provides the solution minimizing the cable lengths, preventing the cables to become slack. 


\section{WRENCH AND TWIST FEASIBLE WORKSPACES}

\section{A. Wrench Feasible Workspace}

The platform of the CDPR is modelled as a rigid body attached to $m$ cables. Each cable transmits on the platform a wrench, proportional to the unit wrench $\hat{\mathbf{w}}_{i}$. The cable wrenches, $\mathbf{w}_{i}=\hat{\mathbf{w}}_{i} \tau_{i}, i=1, \ldots, m$, balance the external wrench acting on the platform. We assume the external wrench acting on the platform is bounded according to (7) and (8). These boundaries are provided by the CDPR designer according to the task requirements. The set of external wrenches that the platform has to bare, the so called Required Wrench Set (RWS), $\left[\mathbf{w}_{e}\right]_{r}$, is a polytope.

Cable tensions, $\tau_{i} i=1, \ldots, m$, are affected as well by some limitations. Due to their non-rigid nature, cable tensions are always non-negative. A lower tension boundary, $\tau_{\text {min }}>0$, can be defined by the user in order to prevent the cables to be slack. The cable tensions present also an upper bound, $\tau_{\max }$, associated to the physical characteristics of the cables and the nominal torque of the motors. Thus, the set of cable tensions which can be transmitted by the cables is defined hereafter as the Available Cable Tension Set (ACTS):

$$
[\boldsymbol{\tau}]_{a}=\left\{\boldsymbol{\tau} \mid \tau_{\min } \leq \tau_{i} \leq \tau_{\max }, i=1, \ldots, m\right\}
$$

Due to the previous constraints, cables can transfer on the platform only a bounded set of wrenches, defined as the Available Wrench Set (AWS), $\left[\mathbf{w}_{e}\right]_{a}$. The AWS can be computed from the ACTS recurring to the equation of the static equilibrium (4). Considering that $\left[\mathbf{w}_{e}\right]_{a}$ is the image of $[\boldsymbol{\tau}]_{a}$ under the linear map represented by the wrench matrix $\mathbf{W},\left[\mathbf{w}_{e}\right]_{a}$ is affinely isomorphic to a particular class of polytopes called zonotopes [9], [10].

According to the previous considerations, the static equilibrium of the platform can be assured only if the pose assumed by the platform is wrench feasible. Nominally, a pose is said to be wrench feasible when the CDPR can balance any external wrench $\mathbf{w}_{e}$ included in $\left[\mathbf{w}_{e}\right]_{r}$ according to cable tension limits expressed by $[\boldsymbol{\tau}]_{a}$ :

$$
\forall \mathbf{w}_{e} \in\left[\mathbf{w}_{e}\right]_{r}, \exists \boldsymbol{\tau} \in[\boldsymbol{\tau}]_{a} \text { s.t. }\left\{\begin{array}{l}
\mathbf{w}_{e} \in\left[\mathbf{w}_{e}\right]_{a} \\
\mathbf{W} \boldsymbol{\tau}+\mathbf{w}_{e}=0
\end{array}\right.
$$

From a geometrical point of view, a pose is wrench feasible if $\left[\mathbf{w}_{e}\right]_{r}$ is fully included in $\left[\mathbf{w}_{e}\right]_{a}$ (as shown in Fig.3):

$$
\left[\mathbf{w}_{e}\right]_{r} \subseteq\left[\mathbf{w}_{e}\right]_{a}
$$

The feasibility can be verified according to the procedure proposed by Gouttefarde et al. [11], by measuring the distance between all the vertices of $\left[\mathbf{w}_{e}\right]_{r}$ and the facets of $\left[\mathbf{w}_{e}\right]_{a}$ :

$$
\mathbf{C w}_{e} \leq \mathbf{d}, \quad \forall \mathbf{w}_{e} \in\left[\mathbf{w}_{e}\right]_{r}
$$

The columns of matrix $\mathbf{C}$ represent the facets of $\left[\mathbf{w}_{e}\right]_{a}$ and vector $\mathbf{d}$ the distance of these facets from the origin of the wrench space. Alternative methods for the computation of the WFW can be found in the literature, such as the one provided by Guay et al. [12] and Cruz et al. [13].

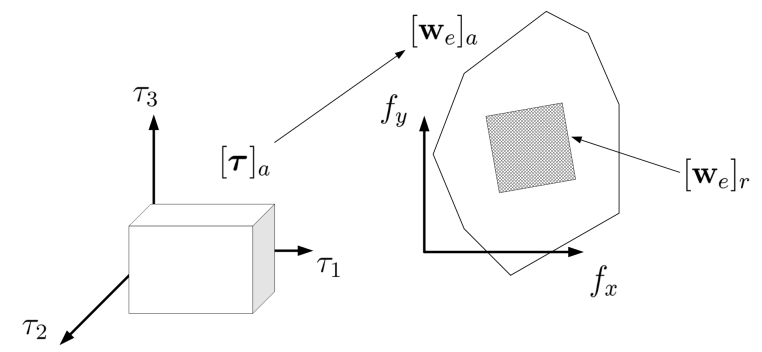

Fig. 3. Example of the mapping of $[\boldsymbol{\tau}]_{a}$ into the wrench space, for a planar CDPR with 3 actuators.

\section{B. Twist Feasible Workspace}

The definition of WFW is based on the linearity of the static equilibrium equation, being $\mathbf{W}$ a linear map between the cable tension space and the space of the external wrench acting on the platform:

$$
\mathbf{w}_{e}=\sum_{i=1}^{m} \hat{\mathbf{w}}_{i} \tau_{i}
$$

The kinematic problem described in (9) presents a similar structure. The geometric Jacobian matrix, $\mathbf{J}$, is a linear map between the space of the cable velocities and the space of the platform twist. As a matter of fact, (9) can be rewritten as a linear combination of the cable twists, $\dot{\mathbf{p}}_{i}, i=1, \ldots, m$ (being $\dot{\mathbf{p}}_{i}, i=1, \ldots, m$ the column vectors of $\mathbf{J}$ ):

$$
\dot{\mathbf{p}}=\sum_{i=1}^{m} \dot{\mathbf{p}}_{i} \dot{l}_{i}
$$

Due to the similarities of the static and kinematic problems, the considerations performed in Sec. III-A can be extended to the kinematics of the CDPR platform.

During the task execution, the platform is supposed to move within a given range of speeds:

$$
\begin{aligned}
& \dot{t}_{\min } \leq \dot{t}_{x}, \dot{t}_{y}, \dot{t}_{z} \leq \dot{t}_{\max } \\
& \omega_{\min } \leq \dot{\phi}, \dot{\theta}, \dot{\psi} \leq \omega_{\max }
\end{aligned}
$$

where $\dot{t}_{x}, \dot{t}_{y}$ and $\dot{t}_{z}$ represent the components of the platform linear velocity, $\dot{\mathbf{t}}$, along $\mathbf{x}_{b}, \mathbf{y}_{b}$ and $\mathbf{z}_{b}$, respectively. $\dot{\phi}, \dot{\theta}$ and $\dot{\psi}$ represent the components of the platform rotational velocity, $\boldsymbol{\omega}$, around $\mathbf{x}_{b}, \mathbf{y}_{b}$ and $\mathbf{z}_{b}$, respectively. $\dot{t}_{\text {min }}$ and $\dot{t}_{\max }$ are the lower and the upper bounds of the linear velocity components. Analogously, $\dot{\omega}_{\min }$ and $\dot{\omega}_{\max }$ are the lower and the upper bounds of the rotational velocity components. The set of twists, $[\dot{\mathbf{p}}]_{r}$, which is limited by the previous boundaries, is defined as the Required Twist Set (RTS).

Similarly to the WFW, matrix $\mathbf{J}$ depends on the platform position and orientation in space. Thus, the TFW is function of the platform pose. The platform twists depends also on the power of the actuators. The nominal speed of the motors limits the cable coiling/uncoiling speed. The maximum coiling speed is defined as $\dot{l}_{\text {max }}$; for most of the actuators, 
considering $i_{\min }=-\dot{l}_{\max }$, the cable speeds are bounded as follows:

$$
i_{\text {min }} \leq i_{i} \leq i_{\max }, \forall i=1, \ldots, m
$$

The set of possible cable coiling/uncoiling speeds is defined hereafter as Available Cable Velocity Set (ACVS), $[\mathrm{i}]_{a}$ :

$$
[\mathbf{i}]_{a}=\left\{\mathbf{i} \mid i_{\min } \leq i_{i} \leq i_{\max }, \forall i=1, \ldots, m\right\}
$$

According to the previous limitations, the platform can assume only a given set of twists, defined hereafter as the Available Twist Set (ATS), $[\dot{\mathbf{p}}]_{a}$. The ATS, which represents a polytope, can be computed from the ACVS using the Jacobian matrix as a linear map from the cable velocity space to the platform twist space.

In order to satisfy the kinematic equilibrium, the CDPR can assume only the poses contained inside the TFW. Nominally, a pose is said to be twist feasible when the CDPR can assume any twist $\dot{\mathbf{p}}$ included in $[\dot{\mathbf{p}}]_{r}$ according to the boundaries of the cable velocities, $[\mathbf{i}]_{a}$ :

$$
\forall \dot{\mathbf{p}} \in[\dot{\mathbf{p}}]_{r}, \exists \dot{\mathbf{l}} \in[\mathbf{i}]_{a} \text { s.t. }\left\{\begin{array}{l}
\dot{\mathbf{p}} \in[\dot{\mathbf{p}}]_{a} \\
\mathbf{J i}-\dot{\mathbf{p}}=0
\end{array}\right.
$$

Alternatively, the twist feasibility condition can be described as:

$$
[\dot{\mathbf{p}}]_{r} \subseteq[\dot{\mathbf{p}}]_{a}
$$

The TFW can be investigated, once again, using to the hyperplane shifting method provided by Gouttefarde $e t$ al. [11]:

$$
\mathbf{C}^{*} \dot{\mathbf{p}} \leq \mathbf{d}^{*}, \quad \forall \dot{\mathbf{p}} \in[\dot{\mathbf{p}}]_{r}
$$

\section{CABLE-DRIVEN PARALLEL ROBOT DESIGN BASED ON THE TASK SPACE ANALYSIS}

\section{A. Description of the actuation and coiling system}

Designing a CDPRs is a complex task which requires the dimensioning of several components and parameters. Most of the research works involving the design of CDPRs are focused on the dimensioning of the platform and the exit point locations. This paper will focus on the dimensioning of:

- The actuators, which in this paper are represented by electric motors, whose nominal torque and speed are defined as $\tau_{M}$ and $\dot{\omega}_{M}$, respectively.

- The winches, whose diameter is equal to $\phi_{w}$.

- The gearboxes, connecting the winches to the motors, whose transmission ratio is equal to $\rho_{R}$.

A scheme of the actuation and transmission system is shown in Fig. 4. Motors limit both the tension transmitted to the cables and the rotational speed of the winches. The gearbox modifies the torque and the speed of the motor according to the following expressions:

$$
\begin{aligned}
\tau_{R} & =\rho_{R} \tau_{M} \\
\dot{\omega}_{R} & =\mu_{R} \dot{\omega}_{M}=\frac{1}{\rho_{R}} \dot{\omega}_{M}
\end{aligned}
$$

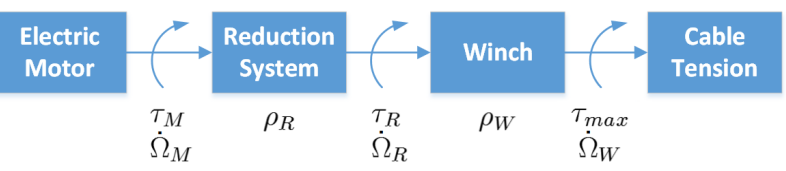

Fig. 4. Scheme of the actuation and transmission system.

The winches are connected to the gearbox. They can transfer on the cable a tension equal to:

$$
\tau_{\max }=\rho_{W} \tau_{R}=\rho_{W} \rho_{R} \tau_{M}=\frac{2}{\phi_{w}} \rho_{R} \tau_{M}
$$

where the winch transmission ratio, $\rho_{W}$, is equal to the inverse of the winch radius, $\rho_{W}=\frac{2}{\phi_{w}}$. Given the winch rotational speed, $\dot{\omega}_{R}=\dot{\omega}_{W}$, the maximum cable speed can be computed as:

$$
i_{\max }=\dot{\omega}_{W} \frac{\phi_{w}}{2}
$$

\section{B. Optimization Problem}

The dimensioning of the motors, the winches and the gearboxes have a great influence on the WFW and the TFW. In order to increase the size of both the workspaces, the design procedure has been formalized as an optimization problem. The user defines the zone of the Cartesian space that the CDPR should attain. This desired zone is discretized into a set of $n_{P}$ points, $P_{k}, k=1, \ldots, n_{P} \in \mathscr{P}$.

The user provides the dimensions of the CDPR platform, as well as the Cartesian coordinates of the exit points of the cables. These informations will be considered as part of the design constant parameters, collected in vector $\mathbf{q}$. The user describes the ACTS and the ACVS, specifying as well the boundaries of the RWS and the RTS.

The objective function of the optimization problem, $\mathscr{V}$, represents the percentage $p_{f}$ of points $P \in \mathscr{P}$ which proofs to be wrench and twist feasible at the same time:

$$
\mathscr{V}(\mathbf{x})=p_{f}=\frac{\sum_{k=1}^{n_{P}} F_{k}}{n_{P}}
$$

where $F_{k}=1$ if the the pose associated to the $k$-th point, $P_{k}$, is both wrench and twist feasible and $F_{k}=0$ otherwise. The design variables are collected in the design variable vector, $\mathrm{x}$ :

$$
\mathbf{x}=\left[\tau_{M}, \dot{\omega}_{M}, \phi_{w}, \rho_{R}\right]
$$

A set of constraints is verified for all the points $P_{k} \in \mathscr{P}$ where the pose assumed by the CDPR is wrench and twist feasible. In this paper, the following constraints are taken into account:

- Cable interferences. This condition is verified by measuring the distance between each pair of cables, using the procedure proposed by Lumelsky in [14]. The interference between the $i$-th cable and the $j$-th cable does not occur when the distance $d_{i, j}^{c c}$ is greater than the diameter of the cables, $\phi_{c}$, i.e.,

$$
d_{i, j}^{c c} \geq \phi_{c} \quad \forall i, j=1, \ldots, m, \quad i \neq j
$$




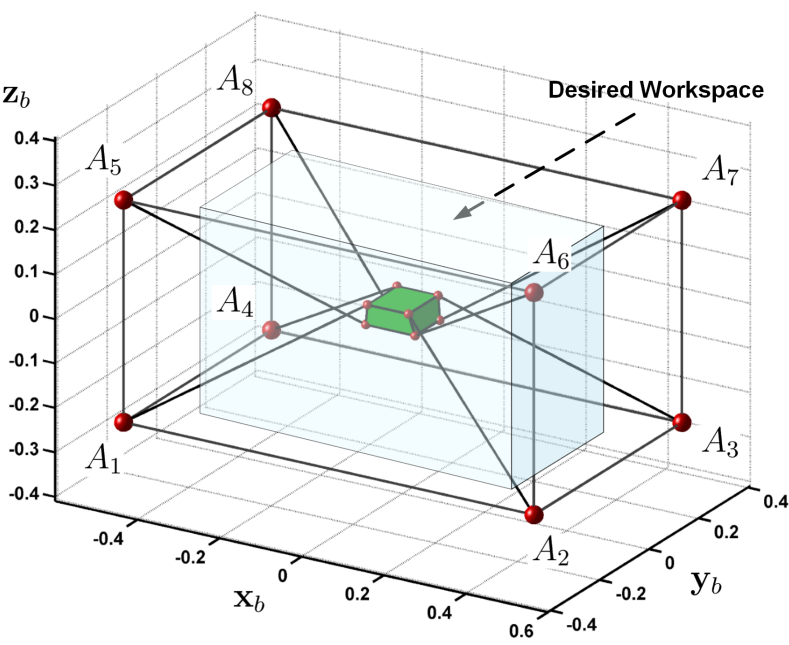

Fig. 5. Description of the case study and the desired workspace.

- Platform positioning error. This constraint is verified by analysing the platform positioning error due to elasticity of the CDPR cables. All the element of the platform positioning error, $\delta \mathbf{t}=\left[\delta \mathbf{t}_{x}, \delta \mathbf{t}_{y}, \delta \mathbf{t}_{z}\right]$, should be lower than a threshold value, $\delta t_{c}$. The platform positioning error can be computed from the CDPR elasto-static model proposed in [15].

The design problem is formulated as follows:

$$
\begin{aligned}
\text { maximize: } & \mathscr{V}(\mathbf{x})=p_{f} \\
\text { over: } & \mathbf{x}=\left[\tau_{M}, \omega_{M}, \phi_{w}, \rho_{R}\right]
\end{aligned}
$$

subject to:

$\forall P_{k} \in \mathscr{P}: F_{k}=1\left\{\begin{array}{l}d_{i, j}^{c c} \geq \phi_{c} \quad \forall i, j=1, \ldots, m, \quad i \neq j \\ -\delta t_{c} \leq \delta t_{x}, \delta t_{y}, \delta t_{z} \leq \delta t_{c}\end{array}\right.$

\section{CASE STUDY}

\section{A. Case Study Description}

The case study presented in this paper is based on the analysis of a spatial CDPR. The robot consists of $m=8$ steel cables. Their diameter, $\phi_{c}$, is equal to $2 \mathrm{~mm}$ and their linear elastic coefficient, $k_{c}$, is equal to $2.52 \times 10^{6} \frac{\mathrm{N}}{\mathrm{m}}$. The cables exit points, $A_{i}, i=1, \ldots, m$, are located at the corner of the base frame, as illustrated in Fig. 5, according to the following Cartesian coordinates:

$$
\begin{aligned}
\mathbf{a}_{1}^{b} & =\frac{1}{2}\left[-s_{l},-s_{w},-s_{h}\right] & \mathbf{a}_{2}^{b} & =\frac{1}{2}\left[s_{l},-s_{w},-s_{h}\right] \\
\mathbf{a}_{3}^{b} & =\frac{1}{2}\left[s_{l}, s_{w},-s_{h}\right] & \mathbf{a}_{4}^{b} & =\frac{1}{2}\left[-s_{l}, s_{w},-s_{h}\right] \\
\mathbf{a}_{5}^{b} & =\frac{1}{2}\left[-s_{l},-s_{w}, s_{h}\right] & \mathbf{a}_{6}^{b} & =\frac{1}{2}\left[s_{l},-s_{w}, s_{h}\right] \\
\mathbf{a}_{7}^{b} & =\frac{1}{2}\left[s_{l}, s_{w}, s_{h}\right] & \mathbf{a}_{8}^{b} & =\frac{1}{2}\left[-s_{l}, s_{w}, s_{h}\right]
\end{aligned}
$$

where the base frame length, $s_{l}$, width, $s_{w}$, and height, $s_{h}$, are equal to $1 \mathrm{~m}, 0.6 \mathrm{~m}, 0.5 \mathrm{~m}$, respectively.

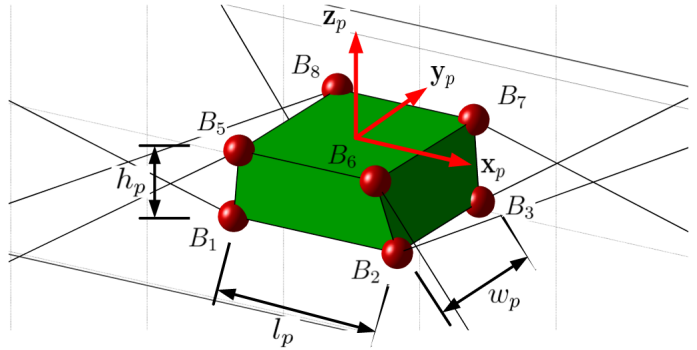

Fig. 6. Platform of the CDPR.

The cables have been connected to the platform according to a cable crossing layout. The main advantages of this layout are the reduction of the cable-cable collisions and the increase of the CDPR stiffness. The Cartesian coordinates $\mathbf{b}_{i}$ of points $B_{i}, i=1, \ldots, m$ are defined as follows:

$$
\begin{aligned}
\mathbf{b}_{1}^{p} & =\frac{1}{2}\left[-l_{p},-w_{p}^{\prime}, h_{p}\right] & \mathbf{b}_{2}^{p} & =\frac{1}{2}\left[l_{p},-w_{p}^{\prime}, h_{p}\right] \\
\mathbf{b}_{3}^{p} & =\frac{1}{2}\left[l_{p}, w_{p}^{\prime}, h_{p}\right] & \mathbf{b}_{4}^{p} & =\frac{1}{2}\left[-l_{p}, w_{p}^{\prime}, h_{p}\right] \\
\mathbf{b}_{5}^{p} & =\frac{1}{2}\left[-l_{p}^{\prime},-w_{p},-h_{p}\right] & \mathbf{b}_{6}^{p} & =\frac{1}{2}\left[l_{p}^{\prime},-w_{p},-h_{p}\right] \\
\mathbf{b}_{7}^{p} & =\frac{1}{2}\left[l_{p}^{\prime}, w_{p},-h_{p}\right] & \mathbf{b}_{8}^{p} & =\frac{1}{2}\left[-l_{p}^{\prime}, w_{p},-h_{p}\right]
\end{aligned}
$$

where $w_{p}^{\prime}=w_{p}+u_{0}$ and $l_{p}^{\prime}=l_{p}+u_{0} . u_{0}$ is an offset applied to the cable-platform connection points in order to avoid cable collisions and singularities. In the specific case, this offset is equal to $1 \mathrm{~cm}$. The platform is a parallelepiped, as shown in Fig. 6. Its width, $w_{p}$, and its length, $l_{p}$, are equal to $10 \mathrm{~cm}$. Its height, $h_{p}$, is equal to $5 \mathrm{~cm}$.

The component of the external forces acting on the platform, $f_{x}, f_{y}$ and $f_{z}$, have been constrained between $\pm 20 \mathrm{~N}$. The components of the external momentum, $m_{x}, m_{y}$ and $m_{z}$, are bounded between $\pm 0.1 \mathrm{Nm}$ :

$$
\begin{aligned}
-20 \mathrm{~N} & \leq f_{x}, f_{y}, f_{z} \leq 20 \mathrm{~N} \\
-0.1 \mathrm{Nm} & \leq m_{x}, m_{y}, m_{z} \leq 0.1 \mathrm{Nm}
\end{aligned}
$$

Analogously, the twist components of the platform, $\dot{t}_{x}, \dot{t}_{y}$ and $\dot{t}_{z}$, have been constrained between $\pm 0.1 \frac{\mathrm{m}}{\mathrm{s}}$. The platform orientation is assumed to be constant, being the frame $\mathscr{F}_{p}$ aligned to the base frame $\mathscr{F}_{b}$.

$$
\begin{gathered}
-0.1 \frac{\mathrm{m}}{\mathrm{s}} \leq \dot{t}_{x}, \dot{t}_{y}, \dot{t}_{z} \leq 0.1 \frac{\mathrm{m}}{\mathrm{s}} \\
\dot{\phi}, \dot{\theta}, \dot{\psi}=0
\end{gathered}
$$

The desired workspace consists of a box, illustrated in Fig. 5. The box has been discretized in $n_{P}=1881$ points, whose coordinates along $\mathbf{x}_{b}, \mathbf{y}_{b}$ and $\mathbf{z}_{b}$ are defined according to the sets of values $[\mathbf{x}],[\mathbf{y}]$ and $[\mathbf{z}]$, respectively:

$$
\begin{aligned}
& {[\mathbf{x}]=\{-0.45 \mathrm{~m}: 0.05 \mathrm{~m}: 0.45 \mathrm{~m}\}} \\
& {[\mathbf{y}]=\{-0.25 \mathrm{~m}: 0.05 \mathrm{~m}: 0.25 \mathrm{~m}\}} \\
& {[\mathbf{z}]=\{-0.2 \mathrm{~m}: 0.05 \mathrm{~m}: 0.2 \mathrm{~m}\}}
\end{aligned}
$$

The range of the design variables, $\tau_{M}, \omega_{M}, \phi_{w}, \rho_{R}$, have been summarized in Tab. I. 
TABLE I

DESIGN VARIABLE BOUNDARIES.

\begin{tabular}{ccccc}
\hline \hline Design Variable & $\tau_{M}$ & $\omega_{M}$ & $\phi_{w}$ & $\rho_{R}$ \\
\hline $\min$ & $0.5 \mathrm{Nm}$ & $50 \mathrm{rpm}$ & $1 \mathrm{~cm}$ & 0.5 \\
$\operatorname{Max}$ & $1.5 \mathrm{Nm}$ & $200 \mathrm{rpm}$ & $5 \mathrm{~cm}$ & 5 \\
\hline
\end{tabular}

\section{B. Solution}

The case study proposed in Sec. V-A has been solved in Matlab using the genetic algorithm toolbox and the GlobalSearch algorithm developed by Zsolt et al. [16].

According to the optimal solution, $57.70 \%$ of points $P_{k} \in \mathscr{P}$ are included in both the WFW and the TFW. The optimal dimensioning of the design variables contemplates a motor nominal torque $\tau_{M}=1.44 \mathrm{Nm}$, a motor nominal speed $\dot{\omega}_{M}=195.6 \mathrm{rpm}$, a winch diameter $\phi_{w}=46.5 \mathrm{~mm}$ and a gearbox transmission ratio $\rho_{R}=3.1$. Fig. 7 represents the intersection of the WFW and the TFW associated to the optimal solution. Under these conditions, the TFW covers the $97.82 \%$ of the desired points $P_{k} \in \mathscr{P}$, as illustrated in Fig. 8; on the contrary, the WFW covers only the $57.73 \%$ of points $P_{k} \in \mathscr{P}$.

The correlation between the design variables, $\mathbf{x}$, and the optimization function, $\mathscr{V}$, has been investigated by means of the Matlab function corrcoef. A set of values has been associated to each design variable:

$$
\begin{aligned}
{\left[\tau_{M}\right] } & =\{-0.5 \mathrm{Nm}: 0.1 \mathrm{Nm}: 1.5 \mathrm{Nm}\} \\
{\left[\omega_{M}\right] } & =\{50 \mathrm{rpm}: 10 \mathrm{rpm}: 200 \mathrm{rpm}\} \\
{\left[\phi_{w}\right] } & =\{1 \mathrm{~cm}: 1 \mathrm{~cm}: 5 \mathrm{~cm}\} \\
{\left[\rho_{R}\right] } & =\{0.5: 0.1: 1,2: 1: 5\}
\end{aligned}
$$

The different combinations of design variables have been tested computing the percentage of feasible points belonging to the WFW and the TFW. As illustrated in Fig. 9, the size of the WFW increases with respect to the nominal torque of the motor, $\tau_{M}$, and the gearbox transmission ratio, $\rho_{R}$. The diameter of the winches, $\phi_{w}$, is inversely proportional to the size of the WFW. Analogously, an increasing of the nominal motor speed, $\dot{\omega}_{M}$, induces an improvement of the TFW size, as well as the increasing of the diameter of the winches. The size of the TFW can be augmented decreasing the the gearbox transmission ratio.

The performed analysis highlights that, regarding the dimensions of both the WFW and the TFW, $\phi_{w}$ and $\rho_{R}$ have an higher influence with respect to the other design variables. Consequently, dimensioning the gearbox transmission ratio and the winch diameter, the designer should take into account the sensibility of these design parameters and the trade-off between the increasing of the WFW size and the decreasing of the TFW size. For example, the largest WFW can be obtained pushing the design variables to their upper limits: $\tau_{M}=1.5 \mathrm{Nm}, \omega_{M}=200 \mathrm{rpm}, \phi_{w}=50 \mathrm{~mm}$ and $\rho_{R}=5$. Under these assumptions, the WFW covers the $65.98 \%$ of $P_{k} \in \mathscr{P}$, as shown in Fig. 10. However, due to the high gearbox transmission ratio, $\rho_{R}=5$, all the points included

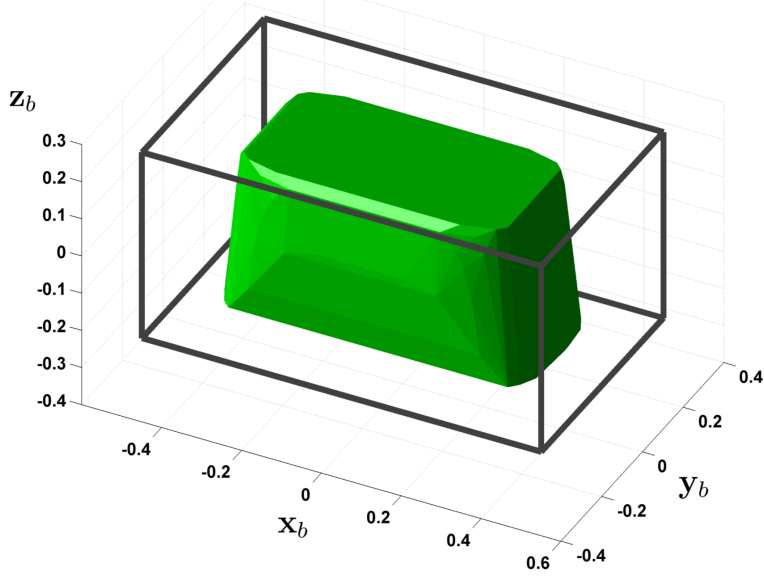

Fig. 7. Intersection of the WFW and the TFW for the optimal solution $\left(\tau_{M}=1.44 \mathrm{Nm}, \dot{\omega}_{M}=195.6 \mathrm{rpm}, \phi_{w}=46.5 \mathrm{~mm}, \rho_{R}=3.1\right)$.

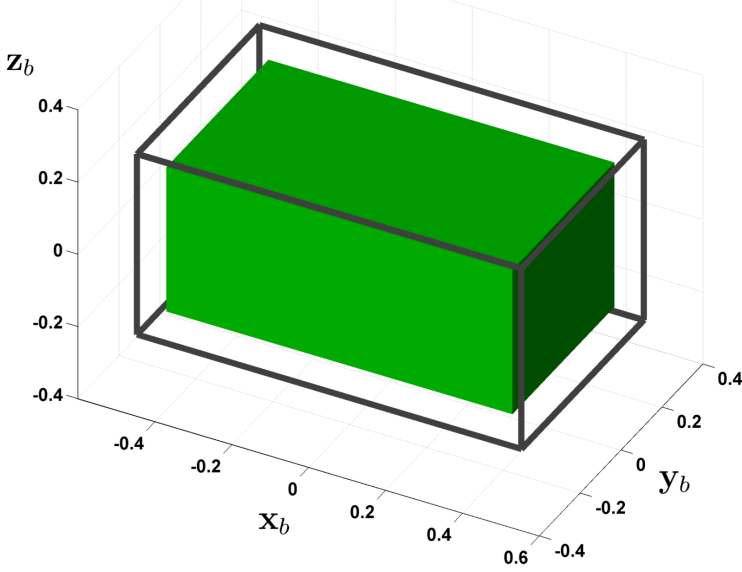

Fig. 8. TFW of the optimal solution $\left(\tau_{M}=1.44 \mathrm{Nm}, \dot{\omega}_{M}=195.6 \mathrm{rpm}\right.$, $\phi_{w}=46.5 \mathrm{~mm}, \rho_{R}=3.1$ ).

in the WFW are not twist feasible.

\section{CONCLUSIONS AND FUTURE WORK}

The research work presented in this paper dealt with the definition of a novel workspace for CDPRs, the Twist Feasible Workspace (TFW). The TFW can be used in order to evaluate the twists that the platform can achieve. Furthermore, the TFW can be successfully integrated in the dimensioning problem of the actuators, the gearboxes and the winches of a CDPR, supposing the locations of the cable exit points and the platform dimensions have been fixed a priori. The influence of the design parameters over the dimensions of the WFW and the TFW have been analysed and commented.

Despite the fact that the computation of the TFW can be performed using methods developed originally to analyse the WFW, part of the research effort should be dedicated to the design of a novel tool for the specific computation of the TFW. Furthermore, more optimization criteria should be 

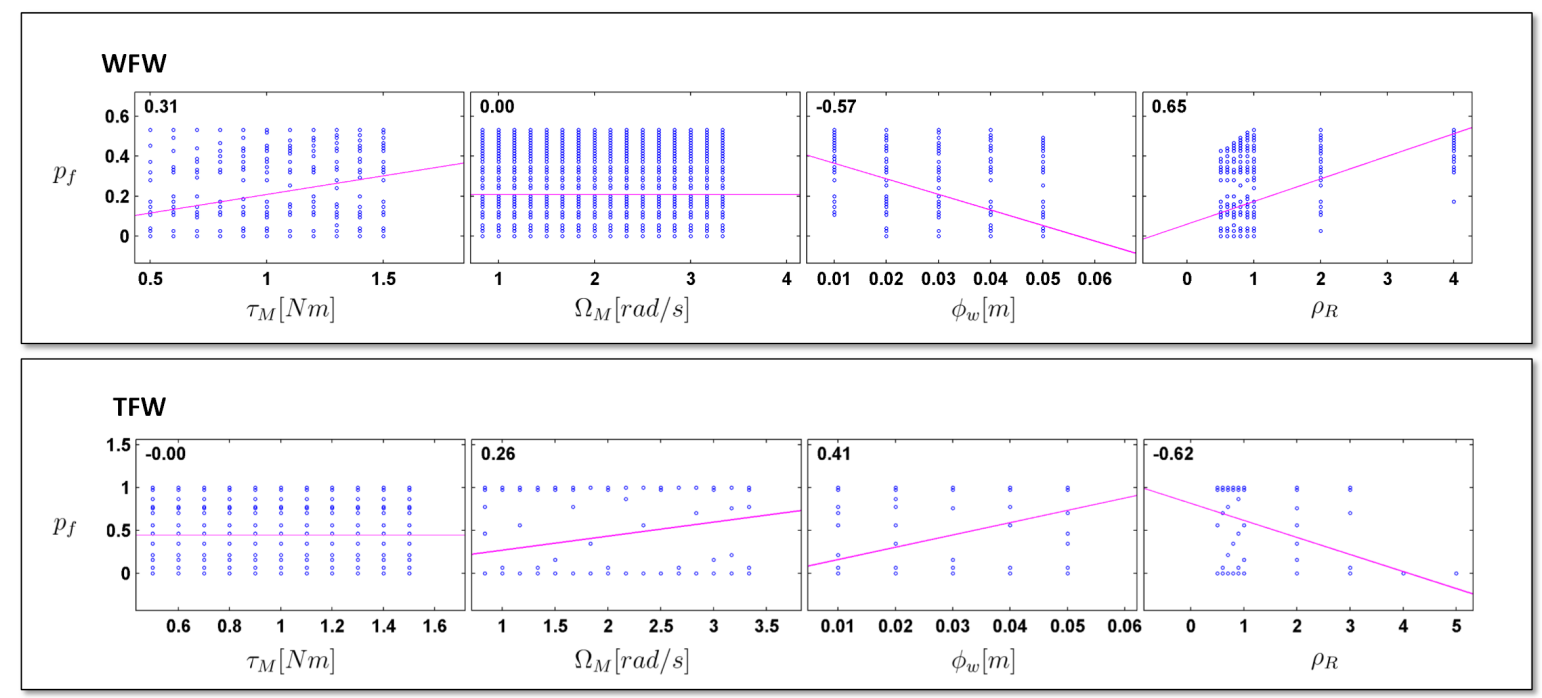

Fig. 9. Analysis of the correlation between the design variables and the optimization criterion.

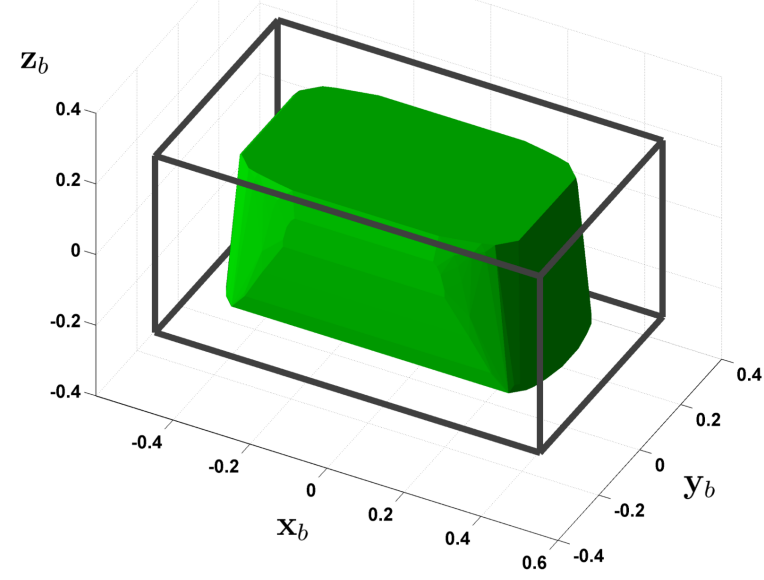

Fig. 10. Larger WFW, covering the $65,98 \%$ of the desired workspace, computed with respect to the design variables $\tau_{M}=1.5 \mathrm{Nm}$, $\dot{\omega}_{M}=200 \mathrm{rpm}, \phi_{w}=50 \mathrm{~mm}, \rho_{R}=5$.

analysed in order to dimension the CDPR components while paying attention to the component costs.

\section{ACKNOWLEDGMENT}

This study is part of the CAROCA project, managed by IRT Jules Verne (French Institute in Research and Technology in Advanced Manufacturing Technologies for Composite, Metallic and Hybrid Structures).

\section{REFERENCES}

[1] A. Pott, C. Meyer, and A. Verl, "Large-scale assembly of solar power plants with parallel cable robots," in International Symposium on Robotics, 2010, pp. 1-6.

[2] C. Holland and D. Cannon, "Cable array robot for material handling," U.S. Patent 6826452, March 2002.

[3] J. Albus, R. Bostelman, and N. Dagalakis, "The NIST spider, a robot crane," J. of Research of the Nat. Inst. of Standards and Technology, vol. 97, no. 3, pp. 373-385, May 1992.
[4] L. Gagliardini, S. Caro, M. Gouttefarde, P. Wenger, and A. Girin, "Optimal design of cable-driven parallel robots for large industrial structures," in IEEE International Conference on Robotics and Automation (ICRA 2014), Hong Kong, China, May 2014.

[5] _ - "A reconfigurable cable-driven parallel robot for sandblasting and painting of large structures," in Cable-Driven Parallel Robots. Springer International Publishing, 2015, vol. 32, pp. 275-291.

[6] SkyCam [Online]. Available: http://skycam.tv/.

[7] G. Rosati, M. Andreolli, A. Biondi, and P. Gallina, "Performance of cable suspended robots for upper limb rehabilitation," in IEEE 10th International Conference on Rehabilitation Robotics (ICORR), 2007, pp. 385-392.

[8] J.-P. Merlet and D. Daney, "A portable, modular parallel wire crane for rescue operations," in 2010 IEEE International Conference on Robotics and Automation (ICRA), May 2010, pp. 2834-2939.

[9] S. Bouchard, C. M. Gosselin, and B. Moore, "On the ability of a cable-driven robot to generate a prescribed set of wrenches," in Proc. of the ASME 2008 Int. Design Eng. Technical Conf. \& Computers and Information in Eng. Conf., Brooklyn, New York, USA,, Aug. 2008, pp. $1-12$.

[10] G. M. Ziegler, Lectures on Polytopes. Graduate Texts in Mathematics 152. Springer-Verlag, 1994, vol. 152.

[11] M. Gouttefarde and S. Krut, "Characterization of parallel manipulator available wrench set facets," in Advances in Robot Kinematics: Motion in Man and Machine, Piran, 2010, pp. 475-484.

[12] F. Guay, P. Cardou, A. Cruz, and S. Caro, "Measuring how well a structure supports varying external wrenches," in The Second Conference on Mechanisms, Transmissions and Applications, Bilbao, Spain, Oct. 2013.

[13] A. Cruz Ruiz, S. Caro, P. Cardou, and F. Guay, "Arachnis : Analysis of robots actuated by cables with handy and neat interface software," in Mechanisms and Machine Science Volume, 2015, vol. 32, pp. 293305.

[14] V. Lumelsky, "On fast computation of distance between line segments," Information Processing Letters, vol. 21, 1985.

[15] M. Arsenault, "Optimization of the prestress stable wrench closure workspace of planar parallel three-degree-of-freedom cable-driven mechanisms with four cables," in Proc. IEEE Int. Conf. on Robotics and Automation, Anchorage, AK, USA, May 2010, pp. 1182-1187.

[16] Z. Ugray, L. Lasdon, J. Plummer, F. Glover, J. Kelly, and R. Marti, "Scatter search and local nlp solvers: A multistart framework for global optimization," INFORMS J. on Computing, vol. 19, no. 3, pp. 328340, 2007. 